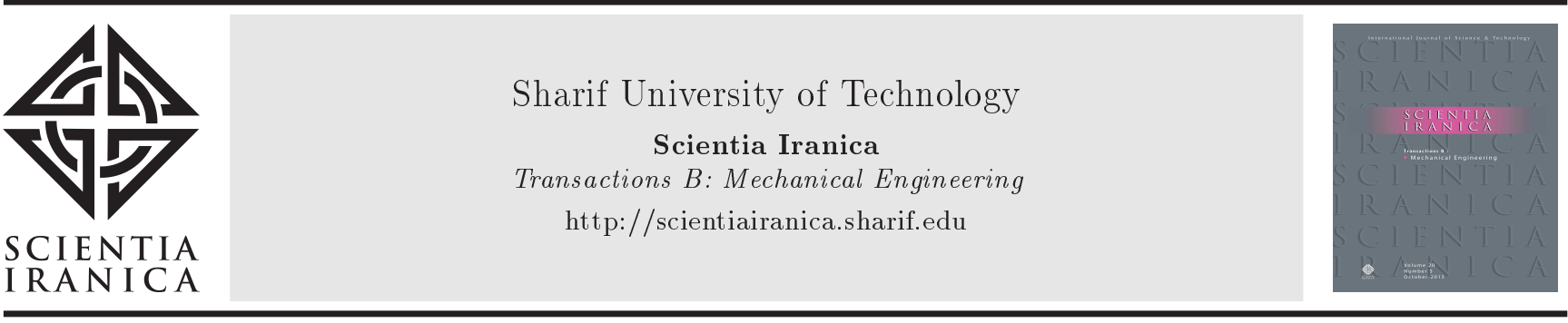

\title{
Dynamic adaptive mesh refinement of fluid-structure interaction using immersed boundary method with two-stage corrections
}

\author{
M.S. Aldlemy ${ }^{a, b, *}$, M.R. Rasani ${ }^{a}$, T.M.Y.S. Tuan ${ }^{c}$, and A.K. Ariffin ${ }^{a}$ \\ a. Department of Mechanical and Materials Engineering, Faculty of Engineering and Built Environment, Universiti Kebangsaan \\ Malaysia, 43600 Bangi, Selangor, Malaysia. \\ b. Department of Mechanical Engineering, Collage of Mechanical Engineering Technology, Benghazi-Libya. \\ c. Department of Mechanical Engineering, Faculty of Engineering, Universiti Teknologi Petronas, 31750 Tronoh, Perak, Malaysia.
}

Received 26 January 2018; received in revised form 6 April 2018; accepted 7 July 2018

\author{
KEYWORDS \\ Two-stage correction; \\ Fluid-structure \\ interaction; \\ Immersed boundary \\ method; \\ adaptive mesh \\ refinement.
}

\begin{abstract}
The application of the Immersed Boundary Method (IBM) coupled with Adaptive Mesh Refinement (AMR) is considered to be one of the powerful tools for solving complex viscous incompressible flow problems. In this paper, the Kajishima-cut-cell IBM is combined with AMR to solve the flow of complex incompressible, viscous fluid, and rigid body problems. In the IBM, the solid and fluid motions at the interface are controlled by a body force that can be calculated with a fraction of solid volume. The objective is to develop an automatic adaptive mesh refinement strategy to enhance the solution in proximity to the fluid-structure interface. This is necessary as the flow field might be significantly affected by the structure boundaries; therefore, it is essential to capture the boundary layers precisely. The capability of this method can be demonstrated through computational results to improve the flow resolution near the fluid structure. The proposed approach is validated using a 2D laminar flow numerical example. The approach is validated in terms of accuracy and performance. The combined IBM-Adaptive mesh refinement approach showed a promising outcome for the investigated problem. The result of the implemented method achieved an acceptable error performance within a reasonably low computation time.
\end{abstract}

(C) 2019 Sharif University of Technology. All rights reserved.

\section{Introduction}

In multi-physics, the Fluid-Structure Interaction (FSI) is a vital, yet challenging, phenomenon that plays a significant path in several engineering and scientific studies. The interaction between a flow field and a deformable or moveable structure is considered in this regard [1]. It is an essential aspect in several engineer-

*. Corresponding author. Tel.: +601114298808 E-mail addresses: maldleme@siswa.ukm.edu.my and maldlemy@ceb.edu.ly (M.S. Aldlemy)

doi: $10.24200 /$ sci. 2018.50347 .1650 ing applications such as bridges, aircraft wings, airbags, turbine blades, and tent roofs. It is also applicable to biological systems such as blood circulation through the vascular system, the movement of air through the pulmonary system, cellular immersion, and the movement of some fish in water [2,3]. It is usually a tough task to develop analytical solutions for modeling FSI problems due to their complex nature. It is also not always easy to conduct experiments on specific problems due to the high cost and the scarcity of the required facilities. Numerical simulations are often considered as a solution to complex problems when investigating the basic phenomenon involved in fluidstructure interactions $[4,5]$. Numerical simulations are 
usually supported by experimental results in many scientific and en gineering fields, thereby eliminating or reducing the need to carry out several experiments. The development of efficient numerical methods and their application for solving complicated FSI problems have attracted much research attention over the years [6,7]. FSI problems can be modeled using a structural mesh, which shares the same attributes with the fluid mesh, or they can be modeled using a structural mesh that bears no structural resemblance to the fluid mesh $[2,8,9]$. The domain and boundaries in the structural mesh approach often change because they follow the path of the interface structure deformation. This requires the use of an Arbitrary Lagrangian-Eulerian (ALE) approach to FSI. Total remeshing or mesh deformation for the whole fluid domain is needed, which requires a reasonable computational effort [10].

In a case where the structural mesh does not conform to the fluid mesh, the Eulerian approach is applied to formulate the fixed fluid grid [7]. This approach is advantageous in that it does not need to continuously update the mesh for the domain. Within this scheme, many approaches have been developed such as the eXtended Finite Element Method (XFEM), which enforces fluid-structure interactions using Lagrangian multipliers [11], a fictitious domain/mortar element method [12], the Immersed Boundary Method (IBM), which interpolates the variables between the fluid and structure using smoothed Dirac-delta function domains [13-15], and the Immersed Finite Element Method (IFEM), which, unlike the IBM method, interpolates the interfacing velocities and body forces using a reproducible kernel particle method [16].

The concept of the IBM was first put forward by [17], in which the impacts of moving boundaries were considered as an external force in the formulation of fluid motion. This was used to satisfy the case of no-slips on the solid boundaries. In another study, the same author and his collaborators developed a deformable thin object that was incorporated into the IBM approach $[18,19]$. IBMs are categorized according to the method of imposing the boundary conditions on the interface. The continuous forcing approach in IBM is normally used for flows that possess immersed elastic boundaries [20]. However, applying this approach to flows with rigid bodies can generate few difficulties. The cut-cell approach in IBM suffers from fluctuations in the calculated value of the pressure.

The fluid structure interaction force in Kajishima's method is modeled using the relative velocities of the phases and a fraction of the solid volume [21]. In this method, there is no loss of momentum because the solid and the fluid share the rectilinear Eulerian grid, thereby ruling out the possibility of interpolation. The variation in the velocity field because of the average volume of the local solid and fluid velocity affects the continuity. The fluid structure interaction in Kajishima's method is coupled with the body force. The inaccurate resolution of the flow near the moving structures is a major disadvantage of the fixed grid approach [22].

The AMR algorithm has been predominantly utilized in the field of fluid-structure en gineering as it is proficient in solving finite element linear and non-linear formulations [23-26]. Based on the AMR concept, a rectangular space was developed by properly nesting grid patches to account for those regions that have steep gradients in the solution, while preserving an acceptable level of error with minimum consumption of time. The adaptive mesh refinement method concentrates on refining certain mesh zones based on the dynamic characteristics of the flow to achieve a suitable grid resolution for any part of the domain [27,28]. This method is beneficial because it is an automatic and dynamic mesh adaptation system that solves flow issues accurately [20]. The fixed mesh construction needs the establishment of the maximum grid resolution in zones that may not be needed for simulation right from the start of the simulation. Most recently, several scholars have established a number of findings in this field. A parallel scheme of an adaptive refinement mesh was investigated for the AMSS-NCKU problem [29]. A stabilized IBM approach, developed for compressible Navier-Stokes equations, was studied by [30]. Another study by [31] was based on GPU-accelerated AMR for the IBM. However, to overcome existing drawbacks, the study is still in the process of developing new methods such as a simple computational process or accurate numerical outcomes.

The interpolation function determines the interaction between the structure and fluid and, also, shapes the space between the structure and fluid interface within the width of the mesh of the fluid grids. It is, however, difficult to capture the resolutions of the boundary layer near the structural surface without using structural body-aligned boundary layer meshes. The disadvantages explained above inspired the development of a numerical technique depending on the integration of adaptive mesh refinement with IBM in a monolithic FSI method, which can solve the interaction problem between a viscous fluid and a rigid body.

In this research, the Fractional Step Method (FSM) can be used to solve the pressure field problem for the thin fluid-solid-interface boundary layers. The equations of motion are implemented in an AMR scheme to achieve a solution that can efficiently capture the structure of flows from such interfaces. This is necessary due to the possibility that the structure can affect the flow field significantly; therefore, it becomes necessary to detect and capture the boundary layers accurately. The applicability of the approach was tested by applying it to $2 \mathrm{D}$ flow fields with a rigid 
body. A benchmark two-dimensional computational problem has validated the effectiveness of the developed formulation.

\section{Governing equation and numerical methods}

\subsection{Basic governing equations}

The Navier-Stokes (N-S) equations are the governing equations and can be described as given below:

$$
\begin{aligned}
& \nabla \cdot u_{f}=0, \\
& \frac{\delta u_{f}}{\delta t}+u_{f} \cdot \nabla \cdot u_{f}=\frac{-1}{\rho f} \nabla p+v_{f} \nabla \cdot\left[\nabla u_{f}+\left(\nabla u_{f}\right)^{T}\right],
\end{aligned}
$$

where $u_{f}$ represents the velocity of fluid, $p$ reflects the pressure, $\rho f$ is the fluid density, and $v_{f}$ is the fluid kinematic viscosity. The Eulerian variables were arranged by the Cartesian grid. Eqs. (1) and (2) were determined via a second-order finite difference method in space and time. Regarding the cells that were partly occupied by the solid structure, the exchange of momentum at their fluid-structure boundary was proposed to be solved by the IBM method. A brief discussion of this method is presented in the next section.

\subsection{Fluid-solid interaction model and numerical implementation}

The IBM method was successfully used to solve the exchange in momentum at the fluid-solid interface for cells partially occupied by intruders [21]. Briefly, for this method, a velocity field was introduced at the solid-fluid interfaces, where the cells were partially filled with solid particles and solved using the IBM. The velocity field was introduced by averaging the volume of the local, $u_{f}$, and solid $u_{p}$ velocities in each cell.

$$
u=(1-\alpha) u_{f}+\alpha v_{p},
$$

where $\alpha(0 \leq \alpha \leq 1)$ represents a fraction of the cell solid volume, as presented in Figure 1. The particle velocity, $u_{p}$, can be separated into rotating and translating components as $u_{p}=v_{t}+r \times \omega_{p}$. The $v_{t}$ is the objects' translating velocity, $\omega_{p}$ is the objects' angular velocity, and $r$ is the relative position of the object from the centre of gravity to a point within the integral region. The volume fraction is evaluated using the following hyperbolic tangent function:

$$
\begin{aligned}
& \alpha=\frac{1}{2}\left[1-\tanh \left(\frac{\delta s}{\sigma \lambda \Delta}\right)\right], \\
& \lambda=\left|n_{x}\right|+\left|n_{y}\right|, \\
& \sigma=0.05\left(1-\lambda^{2}\right)+0.3,
\end{aligned}
$$

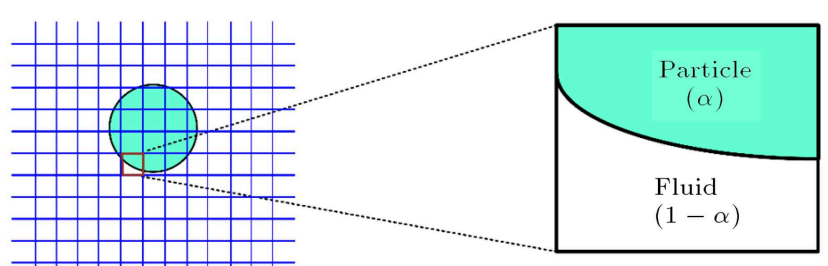

Figure 1. Fluid-solid volumetric fraction for uniform Cartesian cells.

where $n=\left(n_{x}, n_{y}\right)$ is the normal outward unit vector of the surface element, while $\delta_{s}$ is the distance from the elements' central surface. The indicated formulation, called the surface digitizer, was proposed by [32]. Here, the fluid and object interaction could be determined at the interface in the case where the velocity field is in adherence to N-S equations:

$$
\begin{aligned}
\frac{\partial u}{\partial t} & =-\frac{1}{\rho f} \nabla p+H_{u}+f_{p}, \\
H_{u} & =-u \cdot \nabla u+v_{f} \nabla \cdot\left[\nabla u+(\nabla u)^{T}\right] .
\end{aligned}
$$

The following scheme is presented for time advancement for $u$ :

$$
\begin{aligned}
& u^{n+1}=u_{I}-\Delta t \frac{1}{\rho f} \nabla p+\Delta t f_{p}, \\
& u_{I}=u^{n}+\Delta t\left(\frac{3}{2} H_{u}^{n}-\frac{1}{2} H_{u}^{n-1}\right), \\
& f_{p}=\frac{\alpha\left(u_{p}-u_{I}\right)}{\Delta t}
\end{aligned}
$$

where $\Delta t$ is an increase in time, while $f_{p}$ is the intruder effect on the fluid inside the object at the interface. It is worth noting that at $\alpha=0$ with zero interaction force, $f_{p}=0$ is implemented.

On top of that, a second order in accordance with the method presented by Adam Bashforth pertaining to single-phase fluid is employed to define time advancement. The same force, as in Eq. (11), with a reversal sign is applied to the particle fraction in the cell. The body force replaces the hydrodynamic tension at the interface, while the particle phase advancement is completed through the integrated force over the particle volume. Since pressure has been applied to couple the fluid-structure interaction, the numerical schemes are modified. The body force, $f_{p}$, is eliminated from Eq. (7). The following equation reflects the field of velocity in adherence to N-S equations:

$$
\begin{aligned}
\frac{\partial u_{f}}{\partial t} & =-\frac{1}{\rho f} \nabla p+H_{u}, \\
H_{u} & =-u_{f} \cdot \nabla u_{f}+v_{f} \nabla \cdot\left[\nabla u_{f}+\left(\nabla u_{f}\right)^{T}\right] .
\end{aligned}
$$


The following portrays the scheme for time advancement $u_{f}$ :

$$
\begin{aligned}
& u_{I}=u_{f}^{n}+\Delta t\left(\frac{3}{2} H_{u}^{n}-\frac{1}{2} H_{u}^{n-1}\right), \\
& \frac{1}{\rho f} \nabla^{2} p^{n+1}=\frac{\nabla \cdot u_{I}}{\Delta t} \\
& u_{f}^{n+1}=u_{I}-\Delta t \frac{1}{\rho f} \nabla p^{n+1},
\end{aligned}
$$

where $u$ represents the time, $u_{I}$ defines the intermediate velocity, and $\Delta t$ exhibits the increment of the time. Here, the Adam-Bashforth approach is used to determine the time advancement.

\subsection{Two-stage correction of velocity and pressure}

The main idea explained in Eq. (3) involves the solid object being subject to interaction enforcement. Thereafter, the pressure changes were derived. Owing to the fact that the results of the first-order derivation are not corrected with the interaction force enforcement, a second derivation was performed at the affected cells. A small area near the object was selected to correct $u$ in Eq. (3), as denoted by $u_{2}^{n+1}$. In addition, the Simplified Marker and Cell (SMAC) approach was applied to determine the scalar value for the correction of pressure and velocity so as to gain $u_{3}^{n+1}$, and $p_{2}^{n+1}$. In this case, the method of projection was employed to rectify the pressure close to the object surface [33]:

$$
\begin{aligned}
& \frac{1}{\rho f} \nabla^{2} \phi^{n+1}=\frac{\nabla \cdot u_{2}^{n+1}}{\Delta t}, \\
& u_{3}^{n+1}=u_{2}^{n+1}-\Delta t \frac{1}{\rho f} \nabla \phi^{n+1}, \\
& p_{2}^{n+1}=p_{1}^{n+1}+\phi^{n+1},
\end{aligned}
$$

where $u_{3}^{n+1}$ and $p_{2}^{n+1}$ are velocity and pressure, which satisfy the equation of continuity for the following time step.

\section{Adaptive mesh refinement}

In recent times, most algorithms used for integrating hyperbolic equations are equipped with mesh refinement in several ways. The presence of small-scale features and discontinuities in the hyperbolic equations has demanded the application of numerical algorithms to fine meshes with, ideally, smaller scales, compared to those to be solved. On the contrary, an increase in the demand for the computational fluid dynamic capabilities has necessitated the development of algorithms on fine meshes with high-order methods. The computational cost of such issues, including parallel computers, is a major drawback of these methods.

Adaptive mesh refinement based on the grid adaptation technique was first introduced by [28]. The AMR algorithm does not require a considerable decrease in the number of grid cells, but a decrease in the entire number of integrations in the algorithm. Accordingly, this algorithm takes much operational time, particularly for those high-resolution and shock-capturing schemes. AMR can be defined as a combined adaptive method and is two-fold in that an arbitrary grid resolution is allowed by defining a set of overlapping grids through multiple resolutions. For each resolution, the grid hierarchy level is defined only for such a resolution in the part of the foreseen domain. The manner of grid overlapping allows for time refinement, such that, in each grid, there is a temporary step appropriate for its spatial size. To maintain the accuracy and stability of the grids, the time allocated to the grids corresponds to the actual size of their smallest cells. If grids with mixed cell sizes are considered in isolation, coarser cells with bigger time steps could be evolved; however, since, at every iteration, each cell associated with the grid needs to be equally evolved simultaneously, the time step is fixed in the grid for each cell [34]. In the AMR algorithm, fine cells are allowed to evolve with shorter time steps when compared with coarse cells. They are grouped into independent grids, where each group of cells has the same size.

\subsection{Zonal grid refinement and hierarchical refinement}

The use of adaptive schemes has been the first idea for improving the solution near fluid-structure interfaces. This is important because the flow field may be affected by the structure, and it is also necessary to capture the boundary layers accurately. The computations in this paper have been conducted based on the concept of adaptive mesh refinement, which involves the interpolation of nodes into smaller versions. This approach has been developed by computing the stationary flow around a cylinder with a very thin mesh refinement layer. Nodes at intervals of $\Delta X=0.1$ are used to build the initial fluid mesh; the finest final mesh is $80 \times 80(6400)$ with $\Delta x=0.00125$ of fluid nodes mesh. The refinement level in each step along the implicit interface was increased by one. In summary, a generally applicable method for improving the accuracy of fixed grid methods is possible with the finest adaptive mesh refinement surrounding the cylinder. The application of an automatic refinement supports fixed grid methods, which do not support many structural movement predictions. Meanwhile, mesh updates could be frequently conducted in accordance with the implicit interface for transient issues associated with large structural surface movements. It would be beneficial 


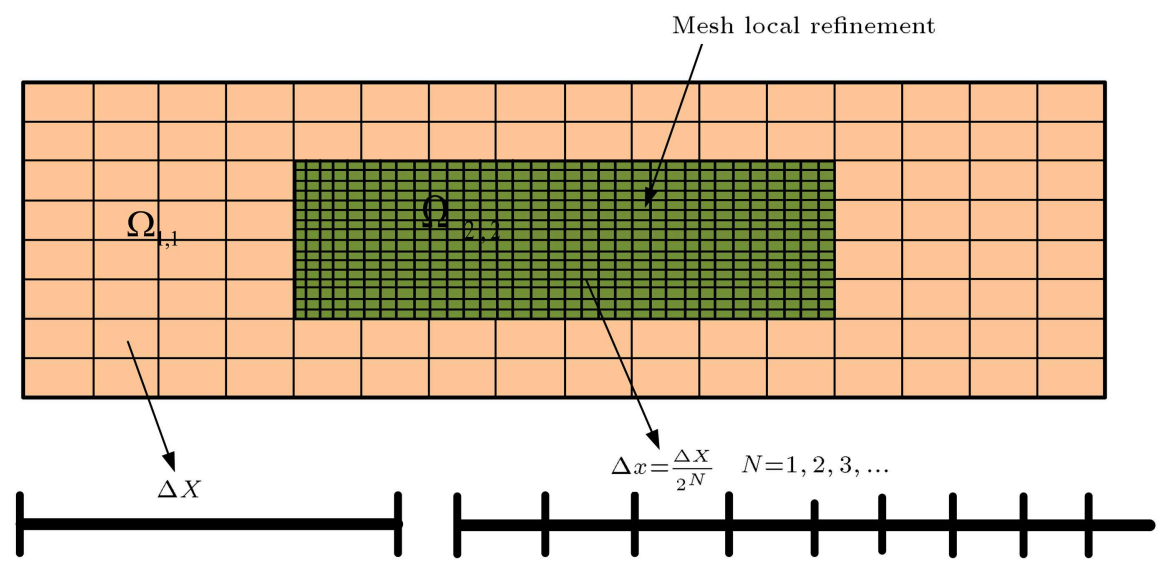

Mesh of grid 1

Mesh of grid 2

Figure 2. AMR for $2 \mathrm{D}$. Refinement is achieved by creating rectangular grid patches with refinement. A finer resolution mesh is applied each time.

in such cases to have a small fluid element layer along the surface of the structure to always keep an optimal boundary layer mesh.

Furthermore, the time variable is considered in accordance with the level of refinement. Mathematically, the time step integration can be expressed in the following term $\Omega_{L e v, k} k=1, \ldots, p_{L e v}$, in which $p_{L e v}$ represents the total number of patches that correlate with the cell size. A synchronization procedure is developed for time step integration among the solution levels. In Figure 2, the grid patches are adjusted in accordance with coarser grid lines or with the rectangular domain boundaries.

There is always a transfer of Eulerian quantities taken to the new hierarchy from the old grid hierarchy; a grid hierarchy is interpolated each time. The refinement formula is $\Delta x=\frac{\Delta X}{2^{N}}$, where $\Delta x$ is the grid size of the new node, $\Delta X$ is the grid size of the old node, and $N$ is the discretizing step. The force densities can also be transported to a new hierarchy from the old one using conservative interpolation and coarsening operators. The fluid pressure is linearly interpolated without any restrictions.

\subsection{Interpolation on the adapted mesh}

The interpolation of all variables at the node level from the old mesh is necessary since it is subject to time variability [35]. This very simple procedure involves the use of a linear interpolation approach, as shown in Figure 3. During the first stage, an initial node, $K_{\text {old }}$, is located "in the rectangular grid of Cartesian" and, then, the new node, $N_{\text {new }}$, is generated for the new mesh. The quantity $N_{\text {new }}$ is obtained here through the local representation regarding $K_{\text {old }}$. Thus, the level set function is anticipated in the continuous space as an advanced step to the interpolation procedure. $K_{\text {old }}$ is located by a particular procedure. The primary node, $N_{1 \text { new }}$, is located through a one-dimensional search in
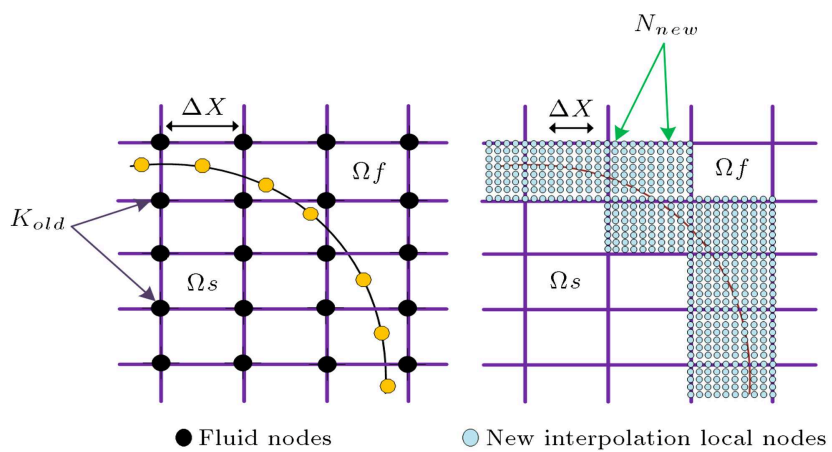

Figure 3. Eulerian and Lagrangian meshes: (left) Fluid-solid interface and (right) computation of interpolation coefficients from locally refines mesh.

accordance with the $K_{1 \text { old }}$ node, in which the search direction is attained through a steep descent algorithm. This is followed by the interpolation of the second node, $N_{1 \text { new }}$, which boards onto the node, $N_{1 \text { new }}$. On the other hand, in searching for the consequent $K_{2 \text { old }}$ initiated from $K_{1 \text { old }}$, this process is performed repeatedly untill the entire new nodes are positioned onto the old mesh. The features of the computational cost regarding search algorithm scales are linear, with $N$ representing the new mesh's nodal number.

A well-known feature of IBM is that the grid lines are not aligned with the surface of the object [13]. Hence, the goal of the immersed boundary method is to estimate the space by assuming that the incompressible solid surface has elastic or visco-elastic properties, as shown in Figure 3. This figure shows that the developed mesh refinement procedure is mostly similar to the generated software modeling, except that the proposed method is simpler and more flexible [36,37].

\subsection{Grid coupling of $A M R$ algorithm and $I B M$}

In the current research, an algorithm was developed 


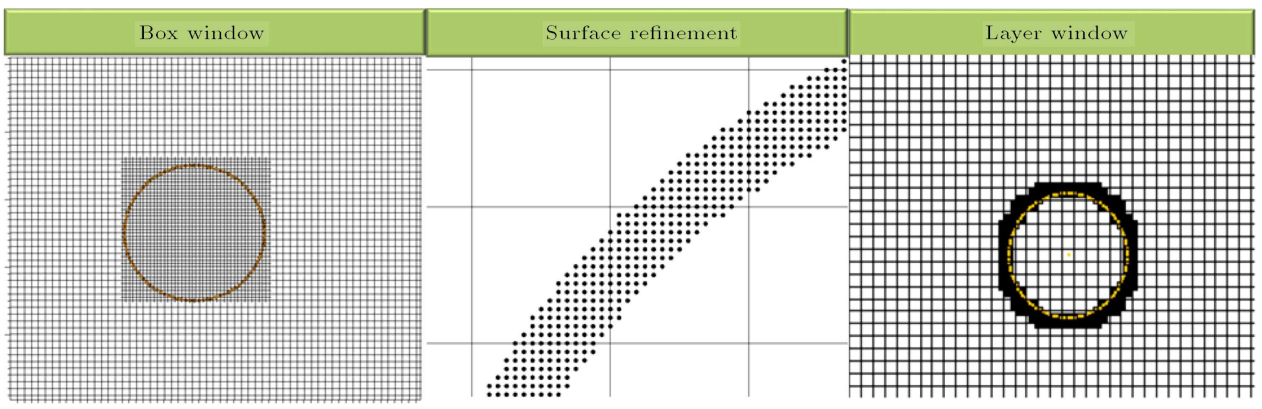

(a)

(b)

(c)

Figure 4. Examples of user-input keywords for local mesh refinement.

1. Solve the $\mathbf{N}-\mathbf{S}$ equations for the whole domain using projection method

2. Compute $d x, d y, d t$ for small box around the solid with respect to the degree of refinement $i$ as, $d x_{\text {new }}=\frac{D X}{2^{i}}, d y_{\text {new }}=\frac{D Y}{2^{i}}, d t_{\text {new }}=\frac{D T}{2^{i}}, i=1,2, \ldots, N$

Check if $i>0$

3. Interpolate $U_{1}, V_{1}, P_{1}$ for the boundaries of the box around the solid.

Else

$$
\text { Define } U P=U 1, P=P 2, V P=V 1
$$

End

4. Calculate the refine mesh node boundary around the solid $U C_{1: N Y+1}^{2: N Y+1}, V C_{1: N Y+1}^{2: N Y+1}$

5. Update the velocity and pressure $u_{1}, v_{1}, p_{1}$ to new values Check if $i>0$

Call CONS to calculate $U_{1}, V_{1}$

Else

Update $U P, V P$

$$
\text { Define } U P=U 1, P=P 2, V P=V 1
$$

End

6. Use (SOABM) and (FSM) to get the intermediate velocity component $u_{1}^{n}, p_{1}^{n}$

7. Apply Successive of relaxation to calculate the pressure using the Poisson equation $u_{2}^{n+1}, p_{1}^{n+1}$

8. Compute the local solid volumetric fraction and update the velocity as eq (3)

9. Use (SMAC) and cell method to find the scalar potential $\phi$ then solve Poisson equation using SOR as eq (17) $u_{2}^{n+1}, \phi^{n+1}$

10. Correct the velocity and pressure $u_{3}^{n+1}, p_{2}^{n+1}$

11. Calculate Adams Bashforth for particle angular $(U p r, V p r)$ and update theta

12. Compute Crank-Nicholson for Move Particles $(X p r, Y p r)$

RETURN TO STEP (1)

Algorithm 1. Monolithic AMR and IBM refined approach within a one-time step.

to control the local resolution in order to generate meshes. Examples of this study are presented in Figure 4. Figure 4(a) shows a box window that enforces the prescribed mesh resolutions inside a box, while Figure 4(b) shows the surface refinement that enforces the prescribed mesh resolution along the surface of the solid body. Figure 4(c) indicates the layer window that enforces a prescribed mesh resolution away from the solid body. To achieve a desired mesh resolution near a solid in IBM, there must be surface refinement. A smooth mesh transition from the boundary to the far field can be achieved with a layer window. The method was developed in open-source software, written in FORTRAN language, and developed based on the Ubuntu 16.04 LTS operating system. The algorithm can be visualized in greater detail (Algorithm 1).

There is a need for optimized sequential, or even parallel, post-processors to manage large datasets that are generated in transient computations to visualize complex flows. One example of such a program is the VisIt 2.12.3 open-source code, which was employed in this work for the visualization of most of the $2 \mathrm{D}$ simulation results. This is a distributed, graphical analysis of and a parallel visualization tool for $2 \mathrm{D}$ defined meshes.

The locally refined mesh surrounding the solid is described in Figure 5. The main feature of this locally refined mesh surrounding the object is its dynamic movement, which does not require much computation since the determination is limited and does not include 


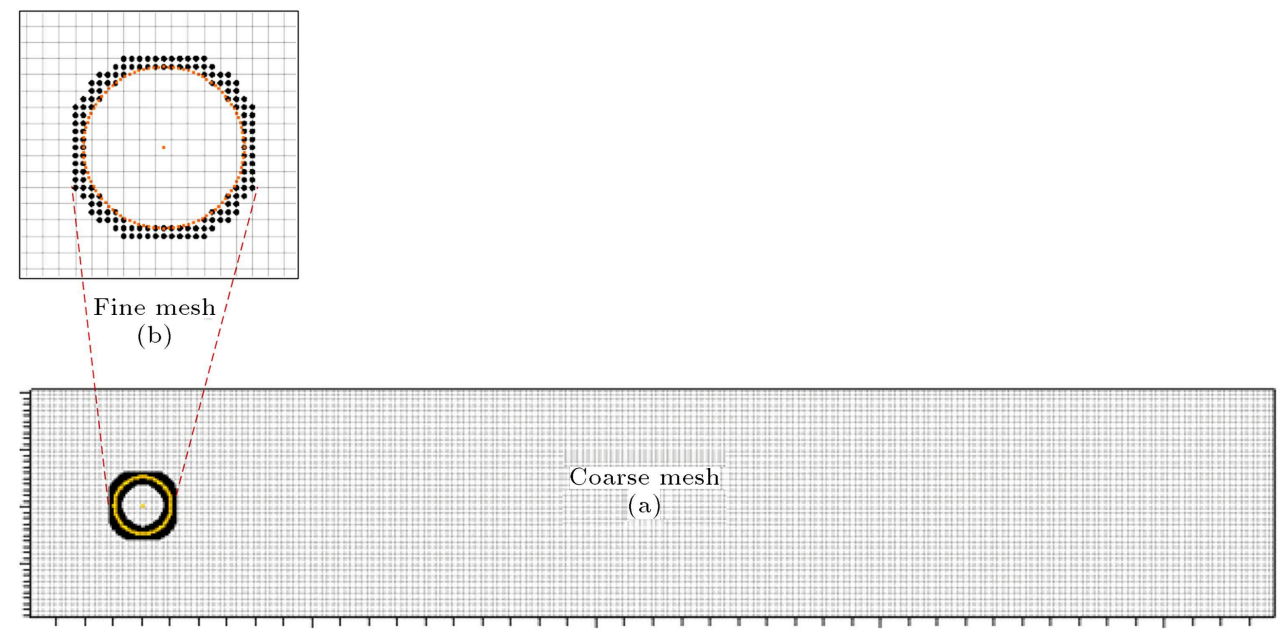

Figure 5. Locally refines mesh for the cylinder.

Table 1. Computational setup for cylinder benchmark.

\begin{tabular}{lcc}
\hline Parameters & Initial mesh (A) & Fine mesh (B) \\
\hline Number of grid point & $220 \times 41$ & $80 \times 80$ \\
Reynolds number $\bar{U} D / \nu$ & 100 & 100 \\
Time increment, $\Delta T$ & 0.0005 & 0.000125 \\
Grid size, $\Delta x, \Delta y$ & 0.01 & 0.0025 \\
\hline
\end{tabular}

the entire domain space. The number of mesh points around the circular cylinder is approximately 6400 nodes with $\Delta t=0.000125 \mathrm{~s}$.

\section{Simulation setup}

A two-dimensional cylinder benchmark background fluid was used as the channel with a width of $2.2 \mathrm{~m} \times$ $0.41 \mathrm{~m}$ and a cylinder radius of 0.05 , located at $(0.2$, 0.2 ). Moreover, prescribed to the channel is a parabolic inflow with $U=1.5 \mathrm{~m} / \mathrm{s}$ and 'no-slip' boundary conditions pertaining to the lower and upper maximum velocities of $U \max x$ walls. The density for the kinematic viscosity was set at $\rho^{f}=1.0 \mathrm{~kg} / \mathrm{m}^{3}$. The Reynolds number obtained was 100 according to the benchmark. For the refined mesh zone, $0.2 \mathrm{~m} \times 0.2 \mathrm{~m}$ is used, the time increment is 0.000125 , and grid size is $\Delta x, \Delta y=0.0025$. Table 1 displays all details of the simulation setup.

\section{Benchmark code validation}

The benchmark research by [38] is recalled here in order to validate the functionality of the developed approach. The numerical value of the laminar flow over a cylinder is equal to 100 . The value of the stationary flow in a two-dimensional case with a diameter $D$ and at different Reynolds numbers has been calculated via $\operatorname{Re}=\bar{U} D / \nu$. For this type of computational modeling, the drag coefficient, $C_{\text {drag }}$, and lift coefficient, $C_{\text {lift }}$, form the interaction simulation. The mathematical formula can be expressed in the following two forms: $C_{\mathrm{drag}}=\frac{2 F_{D}}{\rho U_{m}^{2} D}$ and $C_{\text {lift }}=\frac{2 F_{L}}{\rho U_{m}^{2} D}$. Both coefficients were determined through the integration of the IB force with respect to the Lagrangian space. In addition, the Strouhal number $\mathrm{St}=D f / \bar{U}$ was also computed to validate the cylinder-fluid interaction simulation. The pressure was sourced from the fluid node nearest to the cylinder surface using the described method by employing pressure retrieval. Table 2 shows the result of the lift coefficient and pressure drag coefficient against $\operatorname{Re}=100$. Two batches of results are achieved through the IBM using the rigid cylinder and OpenFOAM-5.0 software. The drag coefficient $\left(C_{\text {drag }}\right)$, lift coefficient $C_{\text {lift }}$, and St from the IBM are shown to be in agreement with the results obtained by the OpenFOAM-5.0 software and with the referenced results.

Table 2. Flow around stationary circular cylinder: $C D$, $C L$, and St for $\operatorname{Re}=100$.

\begin{tabular}{lccc}
\hline Sources & $\boldsymbol{C}_{\text {drag }}$ & $\boldsymbol{C}_{\text {lift }}$ & St \\
\hline Present result & 3.255 & 0.9913 & 0.2997 \\
{$[37]$} & $3.22-3.24$ & $0.990-1.010$ & $0.295-0.305$ \\
{$[39]$} & 3.22583 & 0.98934 & 0.300612 \\
CFD Benchmark & 3.227 & 0.9723 & 0.2985 \\
\hline
\end{tabular}




\section{Numerical results and discussion}

The aim of this work is to develop a mesh refinement technique that is suitable for the interaction of deformable, compressible or incompressible structures with incompressible flows subjected to severe deformations in order to solve FSI problems. The proposed FSI method enjoys two main features: first, it can handle complex and unlimited structural deformations and, second, a proper mesh resolution is preserved around the surface structure that is initially constructed throughout the simulation. These features characterize the proposed method best, making it a proper approach for solving real-world FSI problems. This is further strengthened by the fact that where boundary layers usually exist, there is a layer of meshes where there can be flow separation and re-attachment as the prerequisites for achieving reliable results in the case of complex FSI problems.

In this section, the case of a fluid moving over a solid cylinder using the proposed formulation is discussed. The examined example is a case proposed by [39]. The initial dimensional information provided is that of a rectangular fluid domain with $2.2 \mathrm{~m} \times$ $0.41 \mathrm{~m}$ characterized by a cylindrical diameter of $0.1 \mathrm{~m}$ and positioned at $\left(x_{c}(t=0), 0.22\right)$. The solid cylinder surrounded by a fine layer of mesh is shown in Figure 6. The configuration of the developed model for the mesh refinement has been compared with the benchmark model using OpenFOAM-5.0 software. The visualizations of the two graphs demonstrate that the mesh refinements are similar. The evolution of the lift coefficient and drag coefficient at $\operatorname{Re}=100$ is demonstrated in Figure 7. The figure indicates noticeable agreement between the developed IBM-AMR algorithm and the comparable benchmark model for the lift and drag coefficient over the time-history of the simulation.

The implementation of the developed technique exhibits a very reliable and steady solution for both the pressure and velocity over time. The modeling also demonstrates a very satisfactory level of applicability for the value of the Reynolds number. The left, bottom, and top of the background fluid subdomain boundary are set up at zero velocity. Hence, the

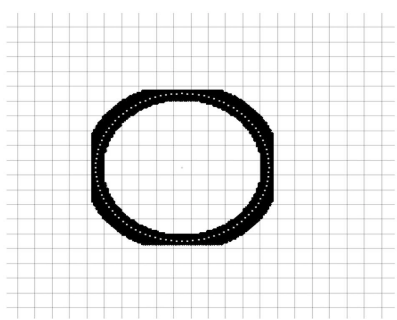

(a) Refinement IBM mesh used in computation

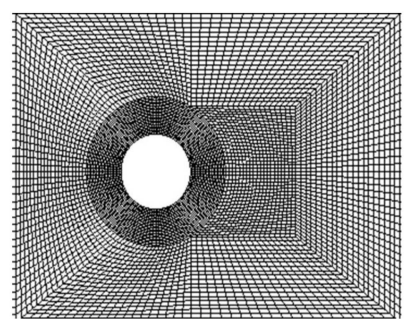

(b) Conventional refinement mesh
Figure 6. Final meshes used for comparison.

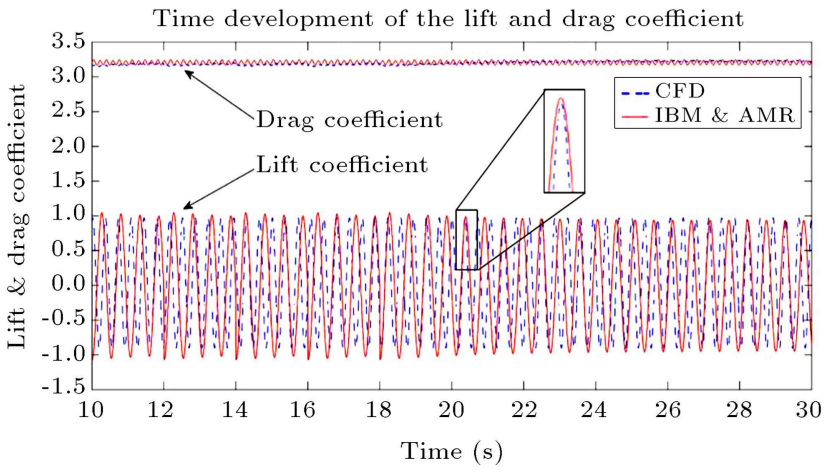

Figure 7. Time-histories of lift and drag coefficients at $\operatorname{Re}=100$.

flow is fixed and bound to the movement of the solid cylinder. The features of the fluid include the viscosity of $\nu=0.001 \mathrm{~m}^{2} / \mathrm{s}$, Reynolds number equal to 100 , and a density of $1.0 \mathrm{~kg} / \mathrm{m}^{3}$. Figure 8 shows that the adaptive refinement mesh for the stationary flow surrounding the cylindrical object is displayed in close visualization for the velocity and pressure indicators. Based on the graphical presentation of these two indicator components, the values are much similar to those determined through modeling using the commercial software, except that the employed algorithm (i.e., monolithic IBM refinement approach with time step) is much simpler, does not require any high-level computations, and is not a time-consuming process. Based on the obtained results, a highly remarkable credit can be given to this algorithm for its wide ranging applications in the case of real-world FSI problems and their analyses such as compressive water flow in or out of pipes and air flow around aeroplane wings causing wing deformation.

The running time for the developed simulation is five seconds with step sizes of one second. The results of the two-dimensional cylinder in terms of velocity and pressure were studied. Figures 9 and 10, respectively, illustrate the velocity and pressure fields in the $x$-direction. The main knowledge and advantage of the current research are that the mesh refinement follows the movement of the object in the fluid space dynamically, which is in contrast to state-of-the-art research [22]. Numerically, the inspected problem is validated with respect to the lift and drag coefficient values, as well as the Strouhal number. Table 2 shows the obtained numerical values and compares them with those of the most reliable studies $[38,40]$ and with the benchmark model.

The calculated computational time for the full refinement mesh simulation is equal to 12531 seconds, although the computational time for the AMR simulation is equal to 1726 seconds, which represents less than $14 \%$ of the full computational time. The results show a very optimistic outcome in comparison to the state- 


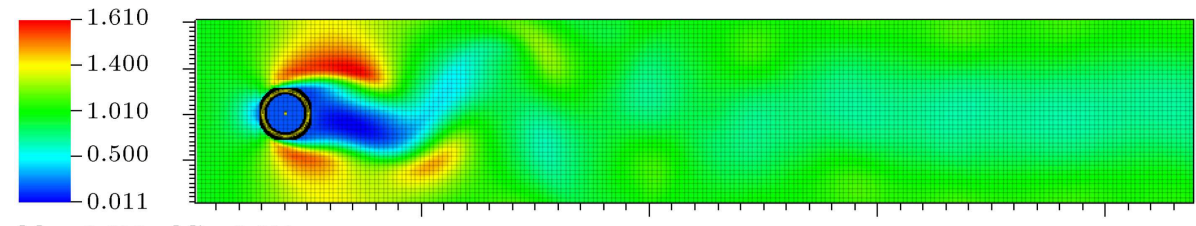

Max: 1.610, Min: 0.011

(a) $x$-component of the velocity field

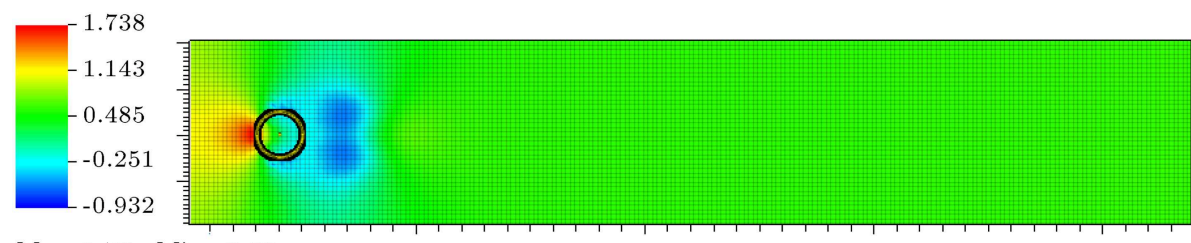

(b) $x$-component of the pressure field

Figure 8. Visualizations of velocity and pressure adaptive refinement for stationary flow around a cylinder. The refinement IBM mesh is displayed in the close-up view: (a) Velocity and (b) pressure fields.
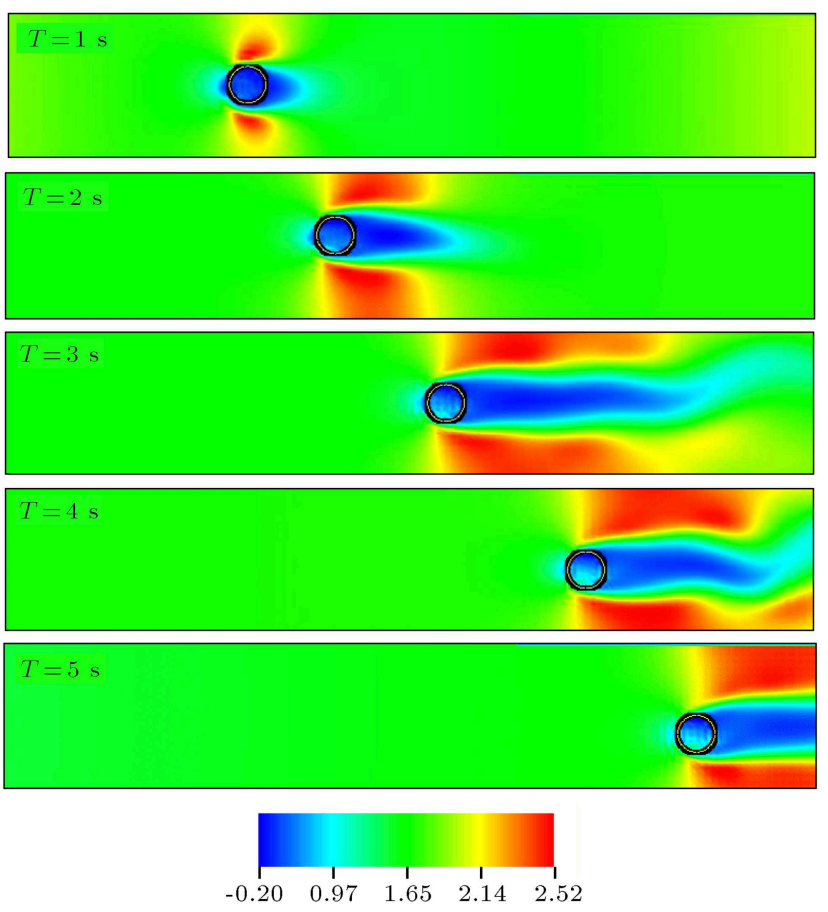

Figure 9. Moving cylinder with refinement dynamic mesh: velocity $(\mathrm{m} / \mathrm{s})$ field in $x, y$ directions at different time steps.

of-the-art study and the benchmark model. However, the advantages of the present research are very simple in that it is less time-consuming and affordable.

\section{Conclusions and remarks}

The simulation of incompressible flows at moving boundaries in a very complex configuration was achieved through the integration of the IBM and AMR. In this paper, a new approach was developed to enhance the solution in proximity to the fluid-solid
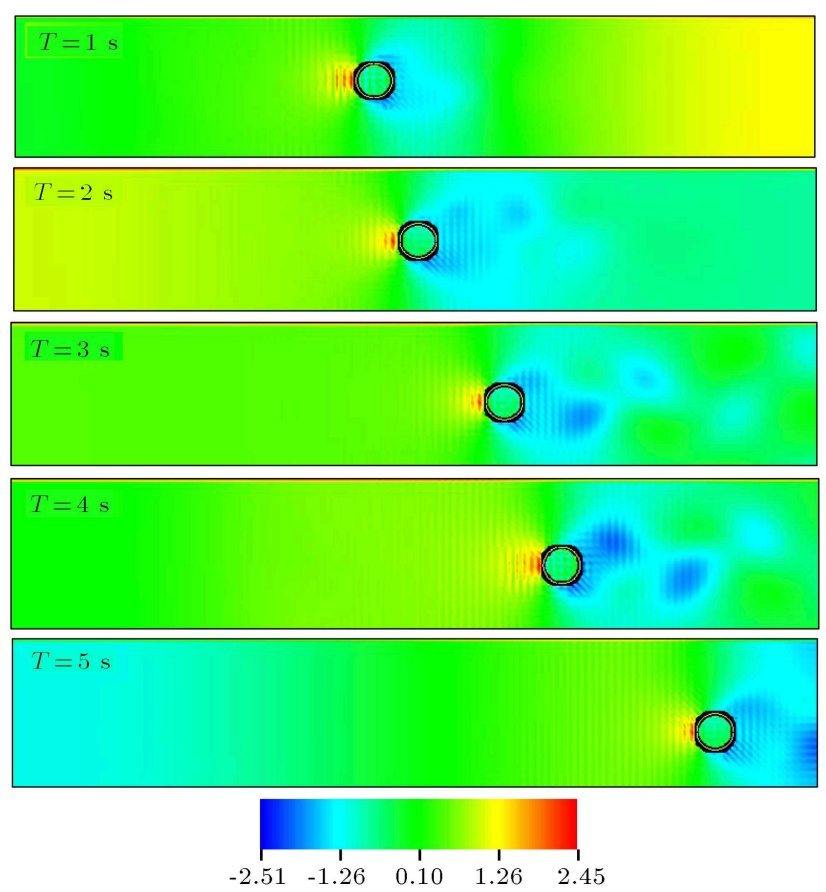

Figure 10. Moving cylinder with refinement dynamic mesh: pressure field at different time steps.

interface for two-dimensional problems. To this end, adaptive mesh refinement was employed in simulation studies to increase the resolution as close as possible towards the solid interface. The time for the integration of the governing equations was accelerated using a relatively coarse mesh with uniform background, which was deployed distant from the fluid-solid interface. Due to the deformability of solid, it has become more desirable to use the AMR algorithm rather than a static body-fitted meshing algorithm. The outcome of the current research exhibited a remarkable performance in comparison to the literature studies. The modeling 
distinguished itself by its simplicity and shorter operation time, compared to soft computing modeling. An extension can be made to new areas that require more resolutions or depend on the properties of the solver. For instance, a thin object analysis can be conducted, as stated in [40].

\section{Acknowledgements}

The authors would like to acknowledge the financial support provided by Ministry of Higher Education (project grant code: FRGS/2/2013/TK01/UKM/ $02 / 2$ ) and the assistance of resources provided at the facility in Faculty of Engineering and Built Environment, Universiti Kebangsaan Malaysia.

\section{References}

1. Zhang, Q. and Hisada, T. "Analysis of fluid-structure interaction problems with structural buckling and large domain changes by ALE finite element method", Comput. Methods Appl. Mech. Eng., 190, pp. 63416357 (2001).

2. Bungartz, H.J. and Schäfer, M.Ed., Fluid-StructureInteraction: Modelling, Simulation, Optimization, Springer Science \& Business Media, 53 (2006).

3. Tezduyar, T.E., Sathe, S., Schwaab, M., and Conklin, B.S. "Arterial fluid mechanics modeling with the stabilized space-time fluid-structure interaction technique", Int. J. Numer. Methods Fluids, 57(5) pp. 601-629 (2008).

4. Gebreslassie, M.G., Tabor, G.R., and Belmont, M.R. "Numerical simulation of a new type of cross flow tidal turbine using OpenFOAM - Part II: Investigation of turbine-to-turbine interaction", Renew. Energy, 50, pp. 1005-1013 (2013).

5. Zhong, J. and Xu, Z. "A reduced mesh movement method based on pseudo elastic solid for fluid-structure interaction", Proceedings of the Institution of $\mathrm{Me}$ chanical Engineers Part C: Journal of Mechanical Engineering Science, 232(6), pp. 973-986 (2018).

6. Costarelli, S.D., Garelli, L., Cruchaga, M.A., Storti, M.A., Ausensi, R., and Idelsohn, S.R. "An embedded strategy for the analysis of fluid structure interaction problems", Comput. Methods Appl. Mech. Eng., 300, pp. 106-128 (2016).

7. Farhat, C. and Lakshminarayan, V.K. "An ALE formulation of embedded boundary methods for tracking boundary layers in turbulent fluid-structure interaction problems", J. Comput. Phys., 263, pp. 53-70 (2014).

8. Tezduyar, T.E., Sathe, S., Cragin, T., Nanna, B., Conklin, B.S., Pausewang, J., and Schwaab, M. "Modelling of fluid-structure interactions with the spacetime finite elements: Arterial fluid mechanics", Int. J. Numer. Methods Fluids, 54, pp. 901-922 (2007).
9. Tezduyar, T.E. and Sathe, S. "Modelling of fluidstructure interactions with the space-time finite elements: Solution techniques", Int. J. Numer. Methods Fluids, 54, pp. 855-900 (2007).

10. Anahid, M. and Khoei, A. "Modeling of moving boundaries in large plasticity deformations via an enriched arbitrary Lagrangian-Eulerian FE method", Sci. Iran. Trans. A., J. Civ. Eng., 17, pp. 141-160 (2010).

11. Chessa, J., Smolinski, P., and Belytschko, T. "The extended finite element method (XFEM) for solidification problems", Int. J. Numer. Methods Eng., 53, pp. 1959-1977 (2002).

12. Baaijens, F.P.T. "A fictitious domain mortar element method for fluid-structure interaction", Int. J. Numer. Methods Fluids, 35, pp. 743-761 (2001).

13. Mittal, R. and Iaccarino, G. "Immersed boundary methods", Annu. Rev. Fluid Mech., 37, pp. 239-261 (2005).

14. Kim, J., Kim, D., and Choi, H. "An immersedboundary finite-volume method for simulations of flow in complex geometries", J. Comput. Phys., 171, pp. 132-150 (2001).

15. Zhang, L., Gerstenberger, A., Wang, X., and Liu, W.K. "Immersed finite element method", Comput. Methods Appl., Mech. Eng., 193, pp. 2051-2067 (2004).

16. Peskin, C.S. "Flow patterns around heart valves: A numerical method", J. Comput. Phys., 10, pp. 252271 (1972).

17. Zhu, L. and Peskin, C.S. "Simulation of a flapping flexible filament in a flowing soap film by the immersed boundary method", J. Comput. Phys., 179, pp. 452468 (2002).

18. Zhu, L. and Peskin, C.S. "Drag of a flexible fiber in a 2D moving viscous fluid", Comput. Fluids, 36, pp. 398-406 (2007).

19. Vanella, M., Posa, A., and Balaras, E. "Adaptive mesh refinement for immersed boundary methods", J. Fluids Eng., 136, p. 40901 (2014).

20. Kajishima, T., Takiguchi, S., Hamasaki, H., and Miyake, Y. "Turbulence structure of particle-laden flow in a vertical plane channel due to vortex shedding", JSME Int. J. Ser. B., 44, pp. 526-535 (2001).

21. Wall, W., Gerstenberger, A., and Mayer, U. "Advances in fixed-grid fluid structure interaction", In: ECCOMAS Multidisciplinary Jubilee Symposium, Springer, pp. 235-249 (2009).

22. Vanella, M., Rabenold, P., and Balaras, E. "A directforcing embedded-boundary method with adaptive mesh refinement for fluid-structure interaction problems", J. Comput. Phys., 229, pp. 6427-6449 (2010).

23. Li, S. and Hyman, J.M. "Adaptive mesh refinement for finite difference WENO schemes", Los Alamos Rep, LA-UR- 03-8927 (2003). 
24. Berger, M.J. and Leveque, R.J. "Adaptive mesh refinement using wave-propagation algorithms for hyperbolic systems", SIAM J. Numer. Anal., 35, pp. 22982316 (1998).

25. Li, S. "Comparison of refinement criteria for structured adaptive mesh refinement", J. Comput. Appl. Math., 233, pp. 3139-3147 (2010).

26. Berger, M.J. and Colella, P. "Local adaptive mesh refinement for shock hydrodynamics", J. Comput. Phys., 82, pp. 64-84 (1989).

27. Berger, M.J. and Oliger, J. "Adaptive mesh refinement for hyperbolic partial differential equations", J. Comput. Phys., 53, pp. 484-512 (1984).

28. Löffler, F., Cao, Z., Brandt, S.R., and Du, Z. "A new parallelization scheme for adaptive mesh refinement", J. Comput. Sci., 16, pp. 79-88 (2016).

29. Brehm, C., Hader, C., and Fasel, H.F. "A locally stabilized immersed boundary method for the compressible Navier-Stokes equations", J. Comput. Phys., 295, pp. 475-504 (2015).

30. Ji, H., Lien, F.S., and Zhang, F. "A GPU-accelerated adaptive mesh refinement for immersed boundary methods", Comput. Fluids, 118, pp. 131-147 (2015).

31. Yuki, Y., Takeuchi, S., and Kajishima, T. "Efficient immersed boundary method for strong interaction problem of arbitrary shape object with the self-induced flow", J. Fluid Sci. Technol., 2, pp. 1-11 (2007).

32. Ya, T., Shah, T.M.Y., Takeuchi, S., and Kajishima, T. "Immersed boundary and finite element methods approach for interaction of an elastic body and fluid by two-stage correction of velocity and pressure", In ASME/JSME 2007 5th Joint Fluids Engineering Conference, pp. 75-81 (2009).

33. Baeza, A., Martínez-Gavara, A., and Mulet, P. "Adaptation based on interpolation errors for high order mesh refinement methods applied to conservation laws", Appl. Numer. Math., 62, pp. 278-296 (2012).

34. Zheng, X., Lowengrub, J., Anderson, A., and Cristini, $\mathrm{V}$. "Adaptive unstructured volume remeshing -II: Application to two- and three-dimensional level-set simulations of multiphase flow", J. Comput. Phys., 208, pp. 626-650 (2005).

35. Vanella, M. and Balaras, E. "A moving-least-squares reconstruction for embedded-boundary formulations", J. Comput. Phys., 228, pp. 6617-6628 (2009).

36. Kempe, T. and Frohlich, J. "An improved immersed boundary method with direct forcing for the simulation of particle laden flows", J. Comput. Phys., 231, pp. 3663-3684 (2012).

37. Schäfer, M., Turek, S., Durst, F., Krause, E., and Rannacher, R. "Benchmark computations of laminar flow around a cylinder", In Flow Simulation with HighPerformance Computers II, pp. 547-566 (1996).

38. Codina, R., Houzeaux, G., Coppola-Owen, H., and Baiges, J. "The fixed-mesh ALE approach for the numerical approximation of flows in moving domains", J. Comput. Phys., 228, pp. 1591-1611 (2009).
39. Verkaik, A.C., Hulsen, M.A., Bogaerds, A.C.B., and van de Vosse, F.N. "An overlapping domain technique coupling spectral and finite elements for fluid flow", Comput. Fluids., 100, pp. 336-346 (2014).

40. Chen, X. and Yang, V. "Thickness-based adaptive mesh refinement methods for multi-phase flow simulations with thin regions", J. Comput. Phys., 269, pp. 22-39 (2014).

\section{Biographies}

Mohammed Suleiman Aldlemy is currently a $\mathrm{PhD}$ Candidate at Universiti Kebangsaan Malaysia and an Assistant Lecturer at the Mechanical Engineering Department, Technical College of Mechanical Engineering, Benghazi, Libya. He graduated with a BS in Mechanical Engineering from Higher Institute of Refrigeration and Air-Conditioning Technology, Sukana, Libya and MS in Computational Engineering 2005 from the Department of Mechanical and Materials Engineering, Universiti Kebangsaan Malaysia. His fields of research specialization include computational mechanics, fluid structure interaction, and material engineering.

Mohammad Rasidi Rasani is a Senior Lecturer at the Department of Mechanical and Material Engineering, Faculty of Engineering and Built Environment at the National University of Malaysia. He received his BS degree (in Mechanical Engineering) from the University of New South Wales, Australia in 1996 and an MS degree of science in the same field of study from the National University of Malaysia in 2002. He received his $\mathrm{PhD}$ study in 2012 from RMIT University, Australia. His research interests include fluid-structure interactions, computational fluid dynamics, finite element analysis, and flow-induced energy harvesting.

Tuan Mohammad Yusoff Shah bin Tuan Ya graduated with a BS degree in Engineering in Aeronautical Engineering from University of Salford, UK in 1998. He then joined University Malaya as a tutor and, then, continued his Master in 2000 to pursue Computational Engineering in University of New South Wales, Australia. In 2004, he went to Osaka University, Japan to further his study in $\mathrm{PhD}$. His current researches mainly focus on computational fluid dynamics, finite element analysis, fluid structure interaction, artificial intelligence in fluid dynamics, and multiphase flow.

Ahmad Kamal Ariffin Bin Mohd Ihsan is a Professor at the Department of Mechanical and Materials Engineering, UKM. He graduated with a BS in Mechanical Engineering from UKM in 1990. He then worked as an engineer before joining the Department of Mechanical and Materials Engineering, UKM and 
continued his studies in 1992. End of 1995, he received his $\mathrm{PhD}$ from University of Wales Swansea at the Mechanical Engineering Department and Institute of Numerical Methods in Engineering. Dr. Ahmad Kamal Ariffin teaches mechanics of materials, computational methods in engineering and finite element methods. His specialty lies in the computational method in engi- neering under the area of powder mechanics, fracture mechanics, friction, corrosion, finite element/discrete element, and parallels computations. He is a Fellow at the Institute of Materials Malaysia, founder of Malaysian Association of Computational Mechanics, and also a member of International Association of Computational Mechanics (IACM). 